\title{
A PARTICIPAÇÃO MASCULINA EM GRUPOS DE CONVIVENNCIA NO CONTEXTO RURAL
}

\author{
Alessandra Cardoso Vargas ${ }^{1}$ \\ Marilene Rodrigues Portella ${ }^{2}$
}

resumo

A participação masculina nos grupos de convivência chama a atenção quando é expressiva e se sobressai no que confere ao contexto rural. ○ estudo objetivou identificar os motivos da adesão de homens idosos de uma comunidade rural do norte do estado do Rio Grande do Sul a participar do grupo de convivência e as repercussões percebidas neste processo. Trata-se de uma pesquisa qualitativa descritiva e exploratória cujos dados foram coletados por meio de entrevistas semiestruturadas utilizando a análise temática no tratamento. Os resultados remetem para a importância atribuída pelos sujeitos em compor um grupo a exemplo das experiências existentes no entorno. Exaltam as modificações percebidas no seu viver ao adentrar o grupo de convivência e como este influenciou de forma benéfica nas relações cotidianas, ressaltando os aspectos positivos da trajetória de

1 Graduada em Educação Física (ULBRA). Mestre em Envelhecimento Humano (UPF). E-mail: alessandracvargas@hotmail.com

2 Graduada em Enfermagem (UPF). Doutora em Enfermagem (UFSC). Coordenadora e Docente do Programa de Pós-Graduação em Envelhecimento Humano da Universidade de Passo Fundo - RS.

E-mail: portella@upf.br 
ingresso com destaque para a melhoria das interações, do convívio social e da superação de perdas.

palavras - chave

Grupos de Convivência. Estilo de vida. Sociabilidade.

\section{Introdução}

O envelhecimento demográfico é uma realidade mundial. Na América Latina, países como Brasil, Costa Rica, Equador, México, Panamá e Uruguai confirmam um avanço no processo de envelhecimento populacional, com a característica em que nas áreas urbanas predomina o sexo feminino, ao contrário das áreas rurais, cuja presença masculina se sobressai, e com incremento relativo no grupo de 75 anos e mais como alertou CEPAL (2012) na conferência Regional e Intergovernamental sobre envelhecimento realizada na Costa Rica. Esse fato foi corroborado pelos dados censitários brasileiro ao registrar que a maioria da população de 60 anos ou mais de idade é composta por mulheres (IBGE, 2012).

As transformações demográficas implicam em mudanças quantitativas e qualitativas na forma em que a sociedade se molda e se organiza, além de aportar repercussões em todas as facetas da vida humana, a exemplo da questão social, da composição familiar e da modalidade de convivência, entre outras (CEPAL, 2011).

Uma das formas de inserção e inclusão social da pessoa idosa é a modalidade dos grupos de convivência, também denominados de grupos da terceira idade, em que os participantes reconhecem nesta estratégia o espaço social de lazer, encontros, entre outros. Como descreve Portella (2004), nesses grupos sociais, as pessoas fazem exercícios, usufruem dos momentos de lazer e descontração, adquirem conhecimentos, e trocam ideias e experiências, sendo uma forma de viver com saúde esta etapa da sua vida, lutando contra a estagnação social da velhice.

Entretanto, observa-se nos grupos de convivência que a participação masculina é pouco expressiva. Em parte, a explicação para tal fenômeno pode ser atribuída à forma em que os grupos se organizam em termos de projetos, evidenciando a carência de estratégias motivadoras da inserção masculina nessas práticas sociais (SUZUKI, 2005; MASCARELO; MIORANDO; PORTELLA, 2007). 
Os grupos de convivência de idosos vêm se proliferando em clubes, paróquias, associações comunitárias, centros de saúde e instituições de ensino superior. Em conformidade com estudo de Borges et al. (2008), o perfil indica que os participantes são de baixa renda e independentes para deambulação e para as atividades da vida diária e atividades instrumentais do dia a dia, e relatam satisfação com seus relacionamentos sociais, além do fato de que a grande maioria dos idosos frequentadores confirma a possibilidade de utilização dos grupos de convivência como um cenário que propicia a qualidade de vida.

Neste sentido, o estudo objetivou-se identificar os motivos da adesão de homens idosos de uma comunidade rural do norte do estado do Rio Grande do Sul a participar do grupo de convivência e as repercussões percebidas neste processo.

\section{Decisões metodológicas}

Este estudo é um recorte da pesquisa que deu origem à dissertação de mestrado. Trata-se de um estudo de campo do tipo descritivo/exploratório de caráter qualitativo. Participaram do estudo 34 homens com idade entre 60 e 90 anos que frequentavam o grupo de convivência em uma localidade rural de um município do norte do estado do Rio Grande do Sul. Como critérios de inclusão para a participação desse estudo, levaram-se em conta a adesão livre ao convite e ser frequentador do grupo de convivência há mais de seis meses.

Embora concordando com Spanevello e Matte (2010) que as fronteiras entre o rural e o urbano estão cada vez mais difusas, acentuando-se o domínio do urbano sobre o rural, principalmente devido ao desenvolvimento dos meios de transporte, a penetração do rádio e da televisão, entre outros, neste estudo, considerou-se como meio rural aquele espaço geográfico distanciado do urbano e que, no cenário do estudo, é reconhecido como tal.

Os instrumentos utilizados para a coleta dos dados foram: uma ficha de dados contendo informações sociodemográficos (idade, estado civil, escolaridade, renda mensal e ocupação profissional) e entrevistas semiestruturadas com perguntas abertas (motivo da adesão ao grupo e as repercussões desta inclusão no seu dia a dia). O conteúdo das entrevistas foi registrado em meio eletrônico com anotações em diário de campo para posterior análise.

Segundo Minayo (2007), a análise e a interpretação dentro de uma perspectiva de pesquisa qualitativa não têm como finalidade contar opiniões ou pessoas, pois seu foco é, principalmente, a exploração do conjunto de opiniões e representações sociais sobre o tema que pretende investigar e, deste modo, utilizou-se a proposta da análise temática para tratamento dos dados. 
Atendendo às normas éticas da resolução 196, de 10 de outubro de 1996, do Conselho Nacional de Saúde (CNS)/Ministério da Saúde, que dispõe sobre pesquisas científicas envolvendo seres humanos, aprovado pelo Comitê de Ética em Pesquisa da Universidade de Passo Fundo, protocolo número 0286.0.398.00011, parecer n. 613/2011, os participantes foram esclarecidos detalhadamente a respeito do processo de pesquisa. Sua participação foi formalizada por meio da assinatura do Termo de Consentimento Livre e Esclarecido. A fim de preservar sua identidade, no decorrer dos resultados, suas falas foram identificadas por codinomes utilizando a letra " $\mathrm{E}$ ", significando entrevistado, seguido do número de ordem da classificação das entrevistas.

\section{Análise e discussão}

Com o aumento da expectativa de vida, o homem pode assumir os diversos papéis existentes na sociedade, ocorrendo a possibilidade de ser filho, pai, avô e bisavô, entre outros papéis. Cabe a nós, profissionais da saúde, ter um olhar diferenciado sobre a realidade dos idosos e as estratégias de inserção social deste segmento, e, em especial, sobre a participação masculina nos grupos de convivência, usualmente conhecidos como grupos de terceira idade.

Os dados do presente estudo mostram uma mudança no comportamento do homem idoso. Se antes eram as mulheres que procuram os grupos, agora também são muitos os motivos da adesão masculina.

Nos registros das entrevistas, verificamos que os homens do grupo demonstram aceitar as modificações no organismo que ocorrem com o processo de envelhecimento e aprendem a conviver com estas de maneira muito natural. De acordo com Silva et al. (2007), a população desta geração, ao se confrontar com essas modificações que relacionam-se com o processo de envelhecimento, as compreende como um fato natural e universal.

Evidenciam-se aqui várias razões pelas quais os homens estão inseridos nesse grupo de convivência, no qual muitos foram à procura e outros convidados, pois a necessidade de interagir com outras pessoas, mais especificamente, da mesma geração, é um dos grandes motivos da presença destes no grupo. Pode-se conferir na seguinte fala: É bonito participar [...] é bom [...] fui convidado e entrei, adoro (E32).

Os homens entrevistados relatam que sentem a necessidade de participar de atividades lúdicas com pessoas da mesma geração para que não se sintam sozinhos, pois a participação nelas é uma forma de contribuição nas suas vidas, que vem a somar para a sua existência no contexto social. 
A vivência de uma velhice diferenciada é destacada em suas falas, na qual preservam que a participação nas atividades lúdicas realizadas nos encontros grupais promove a conscientização da população e o reconhecimento em estar reinserido na sociedade em que vivem. Reconhecem, também, o compromisso de saúde como bem-estar individual e coletivo que favorece um envelhecimento bem sucedido, resgatando culturas e papéis sociais que muitas vezes foram perdidos, garantindo-lhes maior autonomia e realização pessoal, e contribuindo para a construção de uma nova mentalidade e realidade social.

Estudos que abordam questões sobre grupos de terceira idade ressaltam que a adesão da pessoa a esta prática social provoca mudanças nos participantes de ordem física, emocional e relacional (FERRIGNO, 2006; PORTELLA, 2004). A mudança física está relacionada à melhora da disposição geral, incluindo a diminuição das dores. No plano emocional, as alterações ficam por conta da melhora do humor e da sensação de um bem-estar subjetivo, já no plano relacional, idosos que passam a participar de grupos estabelecem contatos sociais e significativos, e aumentam o círculo de convivência, além de estabelecer novas amizades.

Como ressalta Portella (2004), se antes os idosos ficavam em casa, cuidando dos netos, fazendo os afazeres domésticos, dentre outras atividades, agora saem desse recinto e passam a frequentar o grupo e, com isso, as oportunidades dos bailes, da atividade física, dos passeios, das viagens e outras tantas ações que lhes tragam satisfação. Neste sentido, observa-se nas falas dos homens o quanto buscam reelaborar o viver do seu dia a dia, construindo meios para se manterem ativos, saudáveis e independentes.

A possibilidade de ampliar o convívio social, assim como o aumento no círculo de amizades somado às viagens realizadas, os encontros com atividades físicas e as horas de lazer vivenciadas entre seus pares, são ações apontadas pelos homens como o fator determinante na continuidade e permanência no grupo de convivência. Segundo Gáspari e Schwartz (2005), os principais motivos que levam alguns indivíduos a buscar esses programas são a busca por diversão, amizades, mudança na rotina, fuga do isolamento, entre outros, como se confere na fala: Olha! Entrei desde início, então a amizade foi o que me incentivo mesmo, porque eu não tinha amigo e nem conhecia ninguém e agora conheço todo mundo (E08).

Ao analisar a fala de E08, percebe-se o quanto o grupo se torna importante para essa população. Chamava atenção a ênfase em que os homens davam ao seu modo de vida rural e à rotina de trabalho. Muitos alegavam ter passado a vida trabalhando de forma exaustiva, enquanto as oportunidades para desfrutar das amizades e diversões eram raras. Assim, hoje com a participação no grupo 
de terceira idade, vislumbram uma nova chance, na qual se prioriza a conquista de novos amigos e a valorização da vida compartilhada entre os demais.

A importância que o grupo exerce na vida dos mais velhos é salientada por Rizzolli e Surdi (2010) ao comentarem que esta possibilidade estimula uma vida social sadia, desenvolve a cultura, favorece os momentos de lazer, eleva a autoestima e, ainda, tais práticas sociais pelo exercício vivenciado se constituem em lições de cidadania (BULLA; SOARES; KIST, 2010).

Além das responsabilidades com o trabalho, a casa e a família, alguns participantes revelam que sua adesão ao grupo, inicialmente, foi motivada pela necessidade de conduzir a esposa. Posteriormente, a obrigação do compromisso é passada à condição de pertença para o casal, pois a oportunidade foi valorizada também pelo fato de propiciar uma melhor socialização, como se confere na fala:

Ah! Isso aí sabe é que nós moremo meio retirado daqui e a minha esposa era uma pessoa que não saia muito, meia parada ai eu vim [...] pra nós participa e eu falei pra ela e ela aceito (EO7).

Na percepção dos homens, suas mulheres, desde muito cedo, têm a função de dedicar-se à família, aos afazeres domésticos e ao cuidado dos entes queridos. Desse modo, ao envelhecer, elas relatam aos homens que "[...] acabam se sentindo sozinhas". Neste seguimento, Bulsing et al. (2007) alertam para o fato que os mais velhos estão encontrando alternativas para reagir e lutar contra o isolamento social. Assim, o grupo atua como um instrumento influente de realização pessoal, estímulo de criatividade e, sobretudo, de reinserção social.

A superação das perdas foi outro dado relevante apontado pelo estudo como uma das razões atribuídas à adesão ao grupo. Os homens deste estudo reconhecem que a interação com seus pares são essenciais para o bem-estar, especialmente para aqueles que passaram por um trauma, tais como a perda de alguém próximo. No entender dos participantes, as atividades grupais devolvem a alegria de viver. Segundo Osório (2007), para além dos processos biológicos e psicológicos, o envelhecimento é um processo cultural e social.

Seguindo essa linha de pensamento, João et al. (2005) salientam que a participação dos idosos nos grupos de convivência pode reestruturar seu papel social, além do forte componente de apoio emocional encontrado nesta inclusão. Para ilustração, observamos a fala que segue:

Antes era eu e minha esposa que faleceu. Minha esposa tinha depressão, quando faleceu eu fiquei muito triste e pior foi que ela se tiro a vida [referindo-se ao suicídio da mesma], todos nós em casa ficamos muito triste, mas fazer o que né. O grupo é distração, é pra te um divertimento né (E12). 
A combinação do sentimento de abandono, solidão e doenças como a depressão são relatadas pela literatura como fatores associados ao risco de suicídio na população idosa (CAVALCANTE; MINAYO; MANGAS, 2012), com profundas repercussões sobre a família. O relato de E12 reforça o papel fundamental dos grupos como uma alternativa de superação das perdas. Neste estudo, a inclusão no grupo de convivência sugere que a tristeza pode ser minimizada pela oportunidade conferida na distração. Segundo Vieira et al. (2011), no século XXI surgem novos contextos e desafios como a passagem da tendência de isolamento social dos idosos para preocupações que envolvem a participação em todas as esferas e dimensões da vida em sociedade.

De fato, neste estudo, observou-se que o grupo de convivência exerce um papel notável na vida dos homens idosos rurais, pois eles encontram, nesta estratégia, possibilidades de uma vida social significativa com o fortalecimento dos laços de amizades e desenvolvimento da sua cultura, além da prospecção dos momentos de lazer. As repercussões se manifestam na melhora da autoestima, no reconhecimento do seu valor social, no compartilhamento de saberes e no exercício da cidadania.

\section{Considerações finais}

Percorrendo o caminho da pesquisa, buscou-se relacionar os motivos determinantes da participação do homem idoso rural no grupo de convivência e as repercussões advindas deste processo. Os resultados remetem para a importância atribuída pelos sujeitos em compor um grupo a exemplo das experiências existentes no entorno. Os participantes expuseram as modificações percebidas no seu viver ao adentrar o grupo de convivência e como este grupo social influenciou de forma benéfica suas relações no cotidiano, ressaltando os aspectos positivos da trajetória de ingresso com destaque para a melhoria das interações, do convívio social e da superação de perdas.

O método adotado mostrou-se apropriado ao estudo cujas entrevistas, para além de uma coleta de dados, se revelaram em momentos de reflexão para os próprios participantes, pois o fato de participarem do processo instigou o olhar sobre a experiência vivenciada. Se, por um lado, a limitação do estudo pode ser conferida a uma realidade específica, que não permite generalizações, por outro, neste mesmo aspecto reside sua contribuição, a especificidade de estudar um público masculino em grupos de convivência, realidade pouco explorada que se recomenda novas investigações. 
A inserção masculina nos grupos de convivência se configura como uma importante alternativa na promoção da saúde no plano individual e coletivo do segmento envelhecente, principalmente quando se pensa em termos de políticas públicas de atenção à pessoa idosa com vistas ao envelhecimento ativo.

\author{
MALE PARTICIPATION IN SUPPORT GROUPS \\ IN THE RURAL CONTEXT
}

abstract

Male participation in support groups calls the attention when it is expressive, is highlighted when it comes to the rural context. The study aimed to identify the reasons for the adherence of elderly men from a rural community in the northern region in the state of Rio Grande do Sul to participate in the support group and the perceived impact during the process. This is a descriptive and exploratory qualitative research from which data were collected through semi-structured interviews using thematic analysis in treatment. The results point to the importance attributed by the individuals to compose such a group of people with existing experiences around. The results exalt the perceived changes in their behavior when entering the support group and how this influenced beneficially in everyday relationships, emphasizing the positive aspects the trajectory of ingress with emphasis on the improvement of social interaction and overcoming mourning.

keywords

Support Groups. Lifestyle. Sociability.

referências

BORGES, Paula Lutiene de Castro; BRETAS, Rose Procópio; AZEVEDO, Silvana Fernandes de; BARBOSA, Juliana Magalhães Machado. Perfil dos idosos freqüentadores de grupos de convivência em Belo Horizonte, Minas Gerais, Brasil. Caderno de Saúde Pública, Rio de Janeiro, v. 24, n. 12, p. 2798-2808, dez. 2008.

BULLA, Leonia Capaverde; SOARES, Erika Scheeren; KIST, Rosane Bernadete Brochier. Cidadania, pertencimento e participação social de idosos - Grupo trocando ideias e matiné das duas: Cine comentado. Ser Social, Brasilia, n. 21, p. 169-196, jul./dez. 2007.

BULSING, Francine Letiele; OLIVEIRA, Kênia Fernandes de; ROSA, Luana Maria Kilian da; FONSECA, Lunara da; AREOSA, Silvia Virginia Coutinho. A influência dos grupos de convivência sobre a autoestima das mulheres idosas do município de Santa Cruz do Sul -RS. Revista Brasileira de Ciências do Envelhecimento Humano, Passo Fundo, v. 4, n. 1, p. 11-17, jan./jun. 2007. 
CAVALCANTE, Fátima Gonçalves; MINAYO, Maria Cecília de Souza; MANGAS, Raimunda Matilde do Nascimento. Diferentes faces da depressão no suicídio em idosos. Revista Ciência e Saúde Coletiva, Rio de Janeiro, v. 46, n. 2, p. 300-309, 2012.

COMISIÓN ECONÓMICA PARA AMÉRICA LATINA Y EL CARIBE. Envejecimiento poblacional. Observatorio demográfico, Santiago de Chile, a. 6, n. 12. CENTRO LATINOAMERICANO Y CARIBEÑO DE DEMOGRAFÍA (CELADE) - División de Población de la CEPAL, Santiago de Chile, 2011.

El envejecimiento demográfico en los censos de la década de 2010. Boletín 10. Envejecimiento y desarrollo en América Latina y el Caribe. CENTRO LATINOAMERICANO Y CARIBEÑO DE DEMOGRAFÍA (CELADE) - División de Población de la CEPAL. Nov. 2012. Disponível em: <http://www.cepal.org/celade>. Acesso em: 15 jun. 2013.

FERRIGNO, José Carlos. Trabalho social com idosos: apresentação da experiência pioneiro do SESC na área do lazer e da cultura. Cadernos de Terapia Ocupacional da UFSCar, São Carlos, SP, v. 14, n. 1, p. 23-31, 2006.

GÁSPARI, Jossett Campagna de; SCHWARTZ, Gisele Maria. O idoso e a ressignificação emocional do lazer. Psicologia: Teoria e Pesquisa, Brasília, v. 21, n. 1, p. 69-76, 2005.

INSTITUTO BRASILEIRO DE GEOGRAFIA E ESTATÍSTICA. Sintese de Indicadores Sociais: uma análise das condições de vida da população brasileira. Estudos e pesquisas de informação demográfica e socioeconômica. Rio de Janeiro, n. 29, 2012. Disponível em: <ftp://ftp.ibge.gov.br/Indicadores_Sociais/Sintese_de_Indicadores_Sociais_2012/ SIS_2012.pdf>. Acesso em: 16 ago. 2014.

JOÃO, Alessandra de Fátima; SAMPAIO, Ângela Andréa Zampieron; SANTIAGO, Elaine Aparecida; CARDOSO, Raquel de Cássia; DIAS, Rosangela Correa. Atividades em grupo: alternativa para minimizar os efeitos do envelhecimento. Revista da Unati, Rio de Janeiro, v. 8, n. 3, p. 397-410, 2005.

MASCARELO, Andréia; MIORANDO, Gláucia Teresinha; PORTELLA, Marilene Rodrigues. Presença masculina nos grupos de terceira idade: o que pensam homens e mulheres sobre a pouca adesão. In: SANTIN, Janaina Rigo; BETTINELLI, Luiz Antônio; BENINCÁ, Ciomara Ribeiro (Org.). Envelhecimento humano: cuidado e cidadania. Passo Fundo: UPF Editora, 2007. p. 204-223.

MINAYO, Maria Cecília de Souza. O desafio do conhecimento: pesquisa qualitativa em saúde. São Paulo: Hucitec, 2007.

OSÓRIO, Agustin Requejo. Os idosos na sociedade atual. In: OSÓRIO, Agustin Requejo; PINTO, Fernando Cabral. (Coord.). As pessoas idosas: contexto social e intervenção educativa. Lisboa: Instituto Piaget, 2007. p. 11-45.

PORTELLA, Marilene Rodrigues. Grupos de terceira idade: a construção da utopia do envelhecer saudável. Passo Fundo: UPF Editora, 2004.

RIZZOLLI, Darlan; SURDI, Aguinaldo César. Percepção dos idosos sobre grupos de terceira idade. Revista Brasileira de Geriatria e Gerontologia. Rio de Janeiro, v. 13, n. 2, p. 225-233, ago. 2010.

SILVA, Carine Alves da; FOSSATTI, Anderlei Fabiano; PORTELLA, Marilene Rodrigues. Percepção do homem idoso em relação às transformações recorrentes do processo de envelhecimento humano. Estudos Interdisciplinares sobre Envelhecimento, Porto Alegre, v. 12, p. 111-126, 2007.

SPANEVELLOS, Rosani Marisa; MATTE, Alessandra. A perspectiva dos pais quanto ao amparo na velhice: um estudo com agricultores familiares sem sucessores. In: ENCONTRO DA REDE DE ESTUDOS RURAIS: MUNDO RURAL, POLÍTICAS PÚBLICAS, INSTITUIÇÕES E ATORES EM RECONHECIMENTO POLÍTICO, 4., 2010, Curitiba. Anais... . Curitiba: UFPR, 2010. CD-ROM.

SUZUKI, Cláudio Shigueki. Aderência à atividade física em mulheres da Universidade Aberta à Terceira Idade. 2005. 1004 f. Dissertação (Mestrado) - Programa de 
Pós-Graduação em Enfermagem em Saúde Pública, Escola de Enfermagem de Ribeirão Preto, Universidade de São Paulo, Ribeirão Preto, 2005

VIEIRA, Péricles Saremba; BAGGIO, André; MARASCHIN, Renata; DAL'VESCO, Francieli. Terceira idade, aposentadoria e grupos de convivência. In: DIEHL, Astor Antônio; VIEIRA, Péricles Saremba; BERTOLIN, Telma Elita (Org.). Envelhecimento humano: experiência, diálogo e conflito. Passo Fundo: UPF Editora, 2011. p. 151-177.

Recebido: 25/09/2013

Aceite Final: 26/11/2014 